\title{
DAS INSTITUT FÜR ASIENKUNDE IN HAMBURG
}

Institut für Asienkunde, 2 Hamburg 36, Alsterglacis 3

ab Sommer 1969: 2 Hamburg 13, Rothenbaumchaussee 32

Fernruf: 458751

Vorsitzender des Vorstandes:

Landgerichtsvizepräsident Dr. Dr. Wilhelm Röhl, Hamburg

Stellv. Vorsitzender:

Prof. Dr. Wolfgang Franke, Hamburg

Institutsdirektor:

Dr. Bernhard Großmann, Hamburg

Vorsitzender des Kuratoriums:

Otto Wolff von Amerongen, Köln

Stellv. Vorsitzender:

Prof. Dr. Herbert Franke, München

Asienkunde ist die wissenschaftliche Beschäftigung mit den Problemen in jenem Raum Asiens, der jenseits der von jeher mit dem Abendlande im Austausch stehenden Ursprungsgebiete des Islam liegt, also mit Süd-, Südost-, Ost- und Zentralasien. Dabei ist es jedoch nicht die Aufgabe der Asienkunde, historische Forschung zu treiben, Stammeskulturen zu untersuchen oder ihr Augenmerk auf Sprachen und Kulturen des genannten Raumes zu richten. Auf diesem Gebiet sind die Universitätsinstitute für Indologie, Sinologie und Japanologie seit vielen Jahren tätig. Vielmehr hat die Asienkunde die Erscheinungen zu untersuchen, die seit der Begegnung mit dem Westen in Asien die Modernisierung ausgelöst haben; und insbesondere die Probleme dieser Modernisierung selbst sind Gegenstand der Asienkunde. Das bedeutet, daß sich die Asienkunde zeitlich auf die neue Geschichte Asiens der letzten hundert Jahre und vor allem auf die Gegenwart beschränkt. Als Zäsur bieten sich dabei in den drei wichtigsten Ländern des genannten Raumes drei Daten an, die man als Ausgangspunkte der Modernisierung bezeichnen kann: die "Mutiny" in Indien (1857/58), der Opiumkrieg in China (1840/42) und die Offnung Japans durch die amerikanische Flotte (1853/54).

Sachlich wird der Gegenstand der Asienkunde insbesondere mit den Methoden der Sozialwissenschaften - also der politischen Wissenschaft, der Soziologie, der Anthropologie, der Rechtswissenschaft und der Ökonomik - untersucht. Daneben tritt die Geographie, die den Lebensraum der asiatischen Menschen beschreibt. Kultur- und Religionswissenschaft, Geschichte und Linguistik sind Hilfswissenschaften der Asienkunde. Aber ohne sie kann es Asienkunde nicht geben. Seriöse wissenschaftliche Arbeit über asienkundliche Probleme ist ohne Landeskenntnis, ohne Sprachkenntnis und ohne das Wissen um kulturelle und historische Zusammenhänge nicht möglich.

Bis etwa 1960 gab es Asienkunde im dargestellten Sinne an keiner deutschen Hochschule. Um jedoch außerhalb der Universitäten die wissenschaftliche Beschäftigung mit dem heutigen Asien zu fördern, wurde auf Grund einer Initiative des Deutschen Bundestages und des Auswärtigen Amtes im Dezember 1956 das Institut für Asienkunde in Hamburg als Stiftung des bürgerlichen Rechts gegründet. Dem Institut ist die Aufgabe gestellt, die heutigen politischen, ökonomischen, sozialen und kulturellen Verhältnisse in Süd-, Südost-, Ost- und Zentralasien wissenschaftlich zu 
erforschen und damit dazu beizutragen, politische Instanzen und die Offentlichkeit der Bundesrepublik Deutschland in ihrem Bemühen zu unterstützen, ein objektives Bild von den Entwicklungen in Asien zu gewinnen. Insbesondere soll das Institut für Asienkunde dabei auch ein Ort sein, an dem sich die in der Form der Sinologie, Japanologie und Indologie an den deutschen Universitäten gepflegten Asienwissenschaften mit den Sozialwissenschaften begegnen.

Als Standort für das Institut für Asienkunde wurde bewußt Hamburg gewählt nicht nur weil der Senat der Freien und Hansestadt Hamburg dem Institut ein Domizil bot, sondern auch weil einerseits die Universität Hamburg ausgezeichnet besetzte und gut ausgestattete Seminare für Sprachen und Kulturen Asiens besitzt, mit denen das Institut eng zusammenarbeitet, und weil andererseits hier das Institut sich auf die Erfahrungen der mehr praxisbezogenen Asienbeziehungen stützen kann, die in dem in Hamburg wirkenden Ostasiatischen Verein institutionalisiert sind. Vorstand und Kuratorium des Instituts setzen sich aus Vertretern der Asienwissenschaft auch außerhalb Hamburgs, des Bundestages und der Wirtschaft, aus Mitgliedern des Hamburger und des Bremer Senats sowie aus Beamten des Auswärtigen Amtes zusammen. Die Mitglieder beider Gremien spiegeln damit gleichzeitig die Sektoren des öffentlichen Lebens wider, in deren Dienst das Institut vor allem tätig ist.

Das Institut für Asienkunde hat seine Arbeit im Frühjahr 1957 mit denkbar kleinem Mitarbeiterstab aufgenommen. Nachdem inzwischen die Beschäftigung mit gegenwartsbezogenen Asienproblemen auch Eingang in die Universitäten gefunden hat, wurde es möglich, weitere Stellen für wissenschaftliche Mitarbeiter einzurichten. Dabei bilden die seit 1965 neben die Zuwendung aus dem Bundeshaushalt tretenden und über das Deutsche Úberseeinstitut in Hamburg geleiteten Haushaltsmittel der Freien und Hansestadt Hamburg eine notwendige und willkommene Ergänzung. Sie ermöglichten es auch, daß das Institut für Asienkunde sich von seinem anfänglichen Schwerpunkt China aus in seiner eigenen Forschungsarbeit auch anderen Regionen Asiens widmen konnte, die vorher ausschließlich von Autoren bearbeitet wurden, die dem Institut fallweise als freie Mitarbeiter zur Verfügung standen. So finden in der eigenen Institutsarbeit neben China insbesondere Japan und Indien Beachtung.

Die China-Abteilung des Instituts wird allerdings in den Jahren 1968-1970 weiter ausgebaut werden. Der Aktualität ihrer Arbeit dienen regelmäßige Forschungs- und Studienaufenthalte der Institutsmitglieder in Ostasien. Eine verstärkte Berücksichtigung Südostasiens ist ebenfalls geplant.

Für die im Institut erarbeiteten bzw. vom Institut in Auftrag gegebenen Forschungsarbeiten stehen folgende Publikationsmöglichkeiten zur Verfügung:

2) "Schriften des Instituts für Asienkunde": die wichtigste Reihe, in der umfangreichere Arbeiten und Dokumentationen sowie Sammelwerke erscheinen. Diese Reihe, die Anfang 1969 die Bandzahl 23 erreichte, wird verlegerisch vom Verlag Otto Harrassowitz, Wiesbaden, betreut.

b) „Mitteilungen des Instituts für Asienkunde“: eine Reihe, die im Eigenverlag des Instituts erscheint und in der kleinere Arbeiten oder Vorträge publiziert werden. Bis Anfang 1969 sind 27 Hefte erschienen.

c) Sonderveröffentlichungen: Größere Arbeiten, die sich ihrem Charakter nach nicht für die beiden genannten Reihen eignen, oder Übersetzungen werden als Sonderveröffentlichungen publiziert.

d) Sonderdruckreihe: Aufsätze von Mitgliedern des Instituts und seiner Gremien, die in den Sachbereich der Institutsarbeit fallen, aber in institutsfremden Publikationen erschienen sind, werden den an der Institutsarbeit interessierten Kreisen durch Aufnahme in die 1968 geschaffene Sonderdruckreihe zugänglich gemacht. Bis Anfang 1969 erschienen sechs Nummern.

e) "Verfassung und Recht in Übersee": An dieser Zeitschrift, die von der Forschungsstelle für Völkerrecht und ausländisches öffentliches Recht der Universität Ham- 
burg und der Hamburger Gesellschaft für Völkerrecht und Auswärtige Politik herausgegeben wird, arbeitet das Institut für Asienkunde neben dem Deutschen Institut für Afrikaforschung und dem Institut für Iberoamerika-Kunde mit.

Die einzelnen Titel sind im Anhang aufgeführt. Auf einige wichtige und für die Arbeit des Instituts kennzeichnende Projekte sei jedoch hier eingegangen:

In einer auf fünf Teilbände angelegten Sammlung (Band XII der „Schriften des Instituts für Asienkunde") veröffentlicht das Institut für Asienkunde eine Dokumentation mit übersetzten Texten der vertraglichen Beziehungen der Volksrepublik China zu anderen Staaten. Eine solche Sammlung erscheint im Institut für Asienkunde erstmalig in der westlichen Welt.

Eine Forschungshilfe, mit der eine bestehende Lücke ausgefüllt werden soll, stellt die kritische Bibliographie dar, die von Frau Dr. Christine Herzer im Jahre 1969 vorgelegt werden wird, in der die wichtigsten Periodika vorgestellt werden sollen, die in den sechziger Jahren über die Volksrepublik China informieren.

In einer dreibändigen Untersuchung zum Thema „Buddhismus, Staat und Gesellschaft in den Ländern des Theravada-Buddhismus" (Band XVII der "Schriften des Instituts für Asienkunde") untersucht Heinz Bechert den oft mißverstandenen politischen Buddhismus in Südostasien.

Ein von Professor Wolfgang Franke betreutes "China-Handbuch", ein Gemeinschaftsprojekt des Instituts für Asienkunde und der diesem eng verbundenen Deutschen Gesellschaft für Ostasienkunde e. V. ist als ein für weite Kreise bestimmtes Nachschlagewerk über das moderne China geplant.

Die Information der Offentlichkeit, die durch Publikationen des Instituts erfolgt, wird ergänzt durch Vortragsveranstaltungen, die das Institut für Asienkunde in Hamburg mit hier ansässigen befreundeten Institutionen durchführt, und durch Vorträge der Mitarbeiter des Instituts in zahlreichen Orten der Bundesrepublik Deutschland.

Das Institut für Asienkunde ist bestrebt, einen lebhaften Kontakt und Austausch $\mathrm{zu}$ anderen Instituten und Organisationen mit ähnlicher Zielsetzung im In- und Auslande zu unterhalten. Über die Deutsche Gesellschaft für Ostasienkunde e. V. in Hamburg und deren Koordinierungsstelle für gegenwartsbezogene Ostasienforschung wie auch über den 1968 gegründeten Arbeitskreis für Forschung und Dokumentation über den süd- und ostasiatischen Raum bestehen laufende Beziehungen zu deutschen Asieninstituten. Das Institut für Asienkunde ist auch Mitglied des Arbeitsausschusses für moderne Chinaforschung der Universität Hamburg. Der Kontakt zur wissenschaftlichen Erforschung der übrigen überseeischen Regionen wird durch die Zusammenarbeit mit dem Deutschen Institut für Afrikaforschung, der deutschen Orient-Stiftung und dem Institut für Iberoamerika-Kunde im Rahmen des Deutschen Übersee-Instituts Hamburg gewährleistet. Im Auslande wurden Kontakte zu verwandten Instituten vor allem in den USA, in England, in den meisten Ländern Asiens und in Australien aufgebaut. Das bedeutende Institute for Developing Economies in Tokyo wählte das Institut für Asienkunde zu seinem Repräsentanten in der Bundesrepublik Deutschland; mit dem Union Research Institute in Hongkong besteht ein Wissenschaftleraustausch.

Neben der Forschungsarbeit ist das Institut für Asienkunde bestrebt, eine auch anderen Instituten und Wissenschaftlern in der Bundesrepublik Deutschland zur Verfügung stehende Quellenbasis aufzubauen. Die Beschaffung der wichtigsten Monographien und Zeitschriftenliteratur in westlichen Sprachen wird dabei vor allem ergänzt durch den Aufbau einer Literatursammlung zu Gegenwartsfragen in ostasiatischen Sprachen. Insbesondere zur Chinaforschung sind im Institut für Asienkunde die wesentlichen Quellen vorhanden. Einzigartig ist in diesem $\mathrm{Zu}$ sammenhang eine Mikrokopie des Archivs des Union Research Institute, das nahezu 3 Millionen durch einen Index zugängliche Zeitungsausschnitte aus den Jahren 1950 bis zur Gegenwart sowie eine große Anzahl seltener Zeitschriften aus der Volksrepublik China umfaßt. Die wichtigste Tageszeitung der Volksrepublik China, 
die „Jen-min Jih-pao“ („Renmin Ribao“), ist vollständig vorhanden. Die ebenfalls vollständigen Ausgaben zweier weiterer wichtiger chinesischer Tageszeitungen aus früheren Jahren, die "Chung-yang Jih-pao“ und die „Ta Kung Pao“, sollen im Laufe des Jahres 1969 angeschafft werden. Eine unentbehrliche Quelle für die gegenwartsbezogene Japanforschung ist die Gesamtausgabe der japanischen Tageszeitung "Asahi Shimbun" (von 1888 bis zur Gegenwart).

Um das im Institut für Asienkunde vorhandene Quellenmaterial zu erschließen und gleichzeitig einen Úberblick über die Mehrzahl der in der Bundesrepublik Deutschland vorhandenen asienkundlichen Publikationen zu schaffen, wird seit 1968 im Institut für Asienkunde eine Leitstelle für die Asiendokumentation aufgebaut, deren Anlauffinanzierung dankenswerterweise von der Stiftung Volkswagenwerk übernommen wurde. Diese Leitstelle arbeitet eng mit den entsprechenden Leitstellen der anderen Hamburger Regionalinstitute zusammen, um in Hamburg eine vor allem von der Deutschen Stiftung für Entwicklungsländer und vom Bundesministerium für wirtschaftliche Zusammenarbeit geförderte Zentraldokumentation des überseeischen Raumes aufzubauen, die angesichts der Fülle des vom einzelnen Wissenschaftler zu bewältigenden Forschungsmaterials notwendig geworden ist, wenn nicht bereits in naher Zukunft eine geordnete Forschungsarbeit durch mühseliges und zeitraubendes Bibliographieren illusorisch werden soll.

\section{Anhang}

Die Publikationen des Instituts für Asienkunde

\section{1. „Schriften des Instituts für Asienkunde“}

Band I Die Verträge der Volksrepublik China mit anderen Staaten (bearbeitet im Institut für Asienkunde)

Frankfurt/M. 1957

Band II T. H. Silcock, O. Schiller, E. S. Kirby, L. Abegg: Studien zur Entwicklung in Südost- und Ostasien Frankfurt/M. 1958

Band III K. Müller, D. E. Groß (Bearb.):

Die wirtschaftliche Verflechtung der Volksrepublik China mit der Sow jetunion

Frankfurt/M. 1959

Band IV Wilhelm Röhl:

Fremde Einflüsse im modernen japanischen Recht

Frankfurt/M. 1959

Band V Jef Last:

Lu Hsün - Dichter und Idol

Ein Beitrag zur chinesischen Geistesgeschichte

Frankfurt/M. 1959

Band VI Klaus Wenk:

Gerichtsverfassung und Zivilprozeß in Thailand

Frankfurt/M. 1960 
Band VII E. J. Solich:

Die UUberseechinesen in Südostasien

Frankfurt/M. 1960

Band VIII D. Jayanama, Klaus Wenk, Max Biehl:

Thailand, Vorträge und Aufsätze

Frankfurt/M. 1960

Band IX Siegfried Böttcher:

Lebensverhältnisse in der japanischen Kleinindustrie

Frankfurt/M. 1961

Band X Hubertus Lupke:

Japans Rußlandpolitik 1939-1941

Frankfurt/M. 1962

Band XI Lily Abegg, Wolfgang Schmahl, Albert Kolb:

Studien zur Entwicklung in Süd- und Ostasien

Neue Folge - Teil 1

Frankfurt/M. 1962

Band XII R. Herzer, W. Mohr, E. Vierheller (Bearb.):

Verträge der Volksrepublik China mit anderen Staaten

Teil 1: Süd- und Ostasien

Frankfurt/M. 1962

Teil 2: Die Länder des Vorderen Orients und Afrikas Frankfurt/M. 1963

Teil 3: Die Länder Westeuropas und Amerikas Frankfurt/M. 1965

Teil 4: Nachträge zu Band XII/1 bis XII/3 Wiesbaden 1968

Band XIII Hemen Ray, Ch'in Mu, W. v. Pochhammer:

Studien zur Entwicklung in Süd- und Ostasien

Neue Folge - Teil 2

Frankfurt/M. 1962

Band XIV W. v. Pochhammer:

Die Auseinandersetzung um Tibets Grenzen

Frankfurt/M. 1962

Band XV Klaus Wenk u. a.:

Thailand-Studien

Frankfurt/M. 1962

Band XVI Dietmar Rothermund, Wolfgang Schmahl, A. A. Bodenstedt, J. A. C. Mackie:

Studien zur Entwicklung in Süd- und Ostasien

Neue Folge - Teil 3: Indonesien

Frankfurt/M. 1964

Band XVII Heinz Bechert:

Buddhismus, Staat und Gesellschaft in den Ländern

des Theravada Buddhismus 
Teil 1: Grundlagen - Ceylon

Frankfurt/M. 1966

Teil 2: Birma, Kambodscha, Laos, Thailand

Wiesbaden 1967

Teil 3: Quellen, Bibliographie, Register

In Vorbereitung

Band XVIII Bernhard Dahm:

Sukarnos Kampf um Indonesiens Unabhängigkeit

Werdegang und Ideen eines asiatischen Nationalisten

Frankfurt/M. 1966

Band XIX Bernhard Großmann (Hrsg.):

Studien zur Entwicklung in Süd- und Ostasien

Neue Folge - Teil 4: Malaysia

Frankfurt/M. 1966

Band XX M. Y. Cho:

Die Entwicklung der Beziehungen zwischen Peking und

P'yongyang 1949-1967

Analyse und Dokumente. Eine Studie über die Auswirkungen des sowjetisch-chinesischen Konfliktes

Wiesbaden 1967

Band XXI Hans Ulrich Storz:

Birma - Land - Geschichte - Wirtschaft

Wiesbaden 1967

Band XXII Paul W. Wilm:

Die Fruchtbarkeit und Ertragsleistung Nordchinas bis 1949

Mit einem Nachwort über den Einfluß der kommunistischen Um-

wälzung auf die Agrarerzeugung des Landes

Wiesbaden 1968

Band XXIII Brunhild Staiger:

Das Konfuzius-Bild im kommunistischen China

Wiesbaden 1969

Band XXIV Sung-Jo Park:

Die Wirtschaftsbeziehungen zwischen Japan und Korea 1910-1968

Wiesbaden 1969

\section{2. „Mitteilungen des Instituts für Asienkunde“}

Heft 1 Werner Levi:

Die Lage in Nepal (vergriffen)

Heft 2 Tilemann Grimm, H. Schulte-Uffelage:

Bericht über das 12. Gespräch der "Junior Sinologues“

in Cambridge/England (vergriffen)

Heft 3 H. Schulte-Uffelage:

Die geschichtlich-geographischen Hintergründe des indisch-

chinesischen Grenzstreites in Ladakh und der North East

Frontier Agency (vergriffen) 
Heft 4 John K. Fairbank:

Einige Grundprobleme der abendländischen Asienforschung (vergriffen)

Heft 5 Heinz Friese:

Der 25. Internationale Orientalistenkongreß in Moskau (vergriffen)

Heft 6 Tilemann Grimm:

Der birmanisch-chinesische Grenzvertrag (vergriffen)

Heft 7 Wolfgang Schmahl:

Indochina - Ein Kapitel französisch-europäischer Kolonialherrschaft

Heft 8 Dick Wilson:

Das Jahr 1960 in Süd- und Ostasien

Heft 9 John K. Fairbank:

China in der amerikanischen Außenpolitik

Heft 10 Institut für Asienkunde:

Die Ansätze zur Verwaltungsreform in Birma

Heft 11 Martin Fischer:

Vierzig Jahre deutsche Chinapolitik

Heft 12 Tilemann Grimm:

Birma nach dem Umsturz

Heft 13 Tilemann Grimm:

Zur Frage der Wiederannäherung zwischen Peking und Moskau 1962 (vergriffen)

Heft 14 Albrecht Magnus:

Zum Verständnis des Japaners in der Gegenwart

1965 (vergriffen)

Heft 15 Hemen Ray:

China und die Länder Südostasiens in der indischen

Außenpolitik

Heft 16 Bernhard Großmann:

Die Asienkunde in den USA (vergriffen)

Heft 17 Wolfgang Bartke:

Die Reisediplomatie der Volksrepublik China (vergriffen)

Heft 18 Fritz Opitz:

Die Ostasienforschung in der Bundesrepublik Deutschland (vergriffen) 
Heft 19 Gudrun Cho:

Zur Frage der diplomatischen Anerkennung der Volksrepublik

China durch die USA 1949-1966

Heft 20 Wolfgang Bartke:

Der diplomatische Dienst der Volksrepublik China

Heft 21 M. Y. Cho:

Die nordkoreanische Führung -

Namensverzeichnis mit Einführung

Heft 22 Die amerikanische Asienpolitik -

Das Reischauer-Hearing vom 31. Januar 1967

Heft 23 Karl Jettmar:

Zur wissenschaftspolitischen Funktion von Außen- und

Kontaktinstituten in Entwicklungsländern

Heft 24 Klaus Röh:

Zum Arbeitsverhalten der indischen Belegschaft in Rourkela

Heft 25 Oskar Weggel:

Die chinesischen Revolutionskomitees

oder

der Versuch, die Große Kulturrevolution durch Parzellierung

$\mathrm{zu}$ retten

Heft 26 Volker Matthies:

Die chinesische Afrikapolitik

Heft 27 Dieter Heinzig:

Die Krise der Kommunistischen Partei Chinas in der Kulturrevolution (geplant)

\section{Sonderveröffentlichungen}

Werner Handke:

Die Wirtschaft Chinas. Dogma und Wirklichkeit

Frankfurt/M. 1959

Kenneth Scott Latourette:

Geschichte des Fernen Ostens in den letzten hundert Jahren

Frankfurt/M. 1959

H. E. Richardson:

Tibet - Geschichte und Schicksal

Frankfurt/M. 1964

Mamoru Shigemitsu:

Die Schicksalsjahre Japans. Vom ersten bis zum Ende des zweiten Weltkrieges 1920-1945

Frankfurt/M. 1959 
Wolfgang Bartke:

Das Politbüro des 8. Zentralkomitees der Kommunistischen Partei Chinas Wiesbaden 1969

\section{Sonderdruckreihe}

Nr. 1 M. Y. Cho:

Die Okinawa-Frage

Nr. 2 Wolfgang Franke:

China und die Dritte Welt

Nr. 3 Wolfgang Franke:

Anpassungsprobleme im chinesischen Erziehungswesen

des 19. und frühen 20. Jahrhunderts

Nr. 4 Tilemann Grimm:

Über das Problem des Weiterwirkens traditioneller Elemente im kommunistischen China

Nr. 5 Wilhelm Röhl:

20 Jahre neues Gerichtswesen in Japan

Nr. 6 Oskar Weggel:

Zentralregierung und Provinzverwaltung auf Taiwan

\section{In Vorbereitung für 1969:}

Australia. A Survey. Edited by V. Venturini.

Siegfried Ebert. Die Aufbauorganisation japanischer Industrieunternehmungen.

Peter Meyer-Dohm. Rajasthan. Die wirtschaftliche Entwicklung eines indischen Staates.

Christine Herzer. Volksrepublik China. Kritische Zeitschriftenbibliographie. 